\title{
Taxonomy and phylogeny of Sidera (Hymenochaetales, Basidiomycota): four new species and keys to species of the genus
}

\author{
Rui Du ${ }^{\text {** }}$ Fang Wu ${ }^{*}$, Genevieve M. Gate², Yu-Cheng Dai', Xue-Mei Tian \\ I School of Ecology and Nature Conservation, PO Box 61, Beijing Forestry University, Beijing 100083, China \\ 2 Tasmanian Institute of Agriculture, Private Bag 98, Hobart, Tasmania 7001, Australia 3 Shandong Provin- \\ cial Key Laboratory of Applied Mycology, Qingdao Agricultural University, Qingdao 266109, China
}

Corresponding author: Yu-Cheng Dai (yuchengd@yahoo.com); Xue-Mei Tian (txm@qau.edu.cn)

Academic editor: K. Hosaka | Received 23 April 2020 | Accepted 10 June 2020 | Published 7 July 2020

Citation: Du R, Wu F, Gate GM, Dai Y-C, Tian X-M (2020) Taxonomy and phylogeny of Sidera (Hymenochaetales, Basidiomycota): four new species and keys to species of the genus. MycoKeys 68: 115-135. https://doi.org/10.3897/ mycokeys.68.53561

\begin{abstract}
Sidera is a polypore genus with white to cream or buff basidiomata, whose species in Hymenochaetales are poorly known. We study the phylogeny and diversity of Sidera based on our recent collections from tropic and subtropic Asian-Pacific regions. Phylogenetic analyses based on the internal transcribed spacer (ITS) and nuclear large subunit (nLSU) ribosomal RNA gene regions indicate that ten terminal lineages are well supported within Sidera. Based on morphological examination and phylogeny, four new species, viz. Sidera minutissima, $S$. parallela, $S$. srilankensis and $S$. tenuis are described, and a new combination, Sidera minutipora, is proposed. All these species are illustrated. Sidera minutissima is characterized by tiny basidiomata with bluish pores when fresh, generative hyphae dominating at the dissepiment edges, the presence of cystidioles, and allantoid basidiospores measuring 3.8-4.4 $\times 0.9-1.3 \mu \mathrm{m}$. Sidera parallela differs from other poroid species in the genus by having parallel tramal hyphae in combination with lunate basidiospores measuring 2.8-3.3 × 0.9-1.2 $\mu \mathrm{m}$. Sidera srilankensis have generative and skeletal hyphae co-dominating at the dissepiment edges, and lunate basidiospores measuring 3.5-4 × 1-1.3 $\mu \mathrm{m}$. Sidera tenuis is distinguished by small pores $(8-10$ per $\mathrm{mm}$ ) and relatively long allantoid basidiospores measuring $4.2-5 \times 0.8-1 \mu \mathrm{m}$. Sidera minutipora is characterized by buff to olivaceous buff basidiomata when dry, $5-7$ pores per $\mathrm{mm}$, rosette-like crystals rare, and allantoid basidiospores measuring 3.7-4.3 $\times 1-1.3 \mu \mathrm{m}$. An identification key to all accepted species is provided.
\end{abstract}

\section{Keywords}

Phylogeny, Rickenellaceae, taxonomy, wood-rotting fungi

* Contributed equally as the first authors.

Copyright Rui Du et al. This is an open access article distributed under the terms of the Creative Commons Attribution License (CC BY 4.0), which permits unrestricted use, distribution, and reproduction in any medium, provided the original author and source are credited. 


\section{Introduction}

Sidera Miettinen \& K.H. Larss. was established by Miettinen and Larsson (2011) based on molecular and morphological analyses, with S. lenis (P. Karst.) Miettinen as the type species. Five species are currently accepted in the genus: S. lenis (= Physisporus lenis P. Karst., Rabenhorst 1886), S. vulgaris (Fr.) Miettinen (= Polyporus vulgaris Fr., Fries 1821), S. lowei (Rajchenb.) Miettinen (= Ceriporiopsis lowei Rajchenb., Rajchenberg 1987), S. lunata (Romell ex Bourdot \& Galzin) K.H. Larss. (= Grandinia lunata Romell ex Bourdot \& Galzin, Bourdot and Galzin 1928), and S. vesiculosa Rui Du \& M. Zhou (Du et al. 2019). The genus is characterized by resupinate, white to cream or buff, mostly waxy basidiomata when fresh, poroid or hydnoid hymenophore, a monomitic or dimitic hyphal system with generative hyphae bearing clamp connections, the presence of rosette-like crystals, and allantoid to lunate basidiospores (Miettinen and Larsson 2011; Du et al. 2019). Species grow on decaying wood and cause a white-rot (Dai et al. 2007; Yuan and Dai 2008; Miettinen and Larsson 2011; Du et al. 2019).

In the phylogeny, current five Sidera species distributed in Europe, Asia, Pacific Ocean and South America were defined based on ITS and nLSU sequences. Sidera vesiculosa, S. lowei, S. vulgaris have distributions in Asian-Pacific regions. However, samples named as Sidera vulgaris from New Zealand and Australia were separated into two lineages (Miettinen and Larsson 2011; Du et al. 2019).

New specimens collected from the tropic and subtropic Asian-Pacific regions have been studied by morphological and DNA methods. As a result, four unknown Sidera species are found. Another species, originally described as Poria minutipora Rodway \& Cleland from Australia, is proposed for transfer to Sidera, and the sample from Australia named as S. vulgaris by Miettinen and Larsson (2011) is also identified as the species. In addition, specimens or literatures and sequences of all ten accepted Sidera species are studied. Furthermore, an identification key to accepted species is provided.

\section{Materials and methods}

\section{Morphological studies}

The studied specimens are deposited at the herbarium of the Institute of Microbiology, Beijing Forestry University (BJFC). Macro-morphological descriptions are based on field notes and dry herbarium specimens. Microscopic measurements and drawings were made from slide preparations of dried specimens stained with Cotton Blue and Melzer's reagent following Dai (2010). In presenting spore size variation, 5\% of measurements were excluded from each end of the range and this value is given in parentheses. The following abbreviations were used: $\mathrm{KOH}=2 \%$ potassium hydroxide, $\mathrm{CB}=$ Cotton Blue, $\mathrm{CB}-=$ acyanophilous, $\mathrm{IKI}=$ Melzer's reagent, IKI $-=$ neither amyloid nor dextrinoid, $\mathrm{L}=$ mean spore length (arithmetic average of all spores), $\mathrm{W}=$ mean spore width (arithmetic average of all spores), $\mathrm{Q}=$ variation in the $\mathrm{L} / \mathrm{W}$ ratios between specimens studied, $\mathrm{n}(\mathrm{a} / \mathrm{b})=$ number of spores $(\mathrm{a})$ measured from given number of 
specimens (b). Special color terms follow Anonymous (1969) and Petersen (1996). Herbarium abbreviations follow Thiers (2018).

\section{Molecular studies}

A CTAB rapid plant genome extraction kit (Aidlab Biotechnologies Co., Ltd., Beijing, China) was used to extract total genomic DNA from dried specimens following the manufacturer's instructions with some modifications (Cui et al. 2019; Shen et al. 2019). ITS regions were amplified with primers ITS4 and ITS5 (White et al. 1990), and the nLSU with primers LR0R and LR7. The PCR procedure for ITS was as follows: initial denaturation at $95^{\circ} \mathrm{C}$ for $3 \mathrm{~min}$, followed by 35 cycles at $94^{\circ} \mathrm{C}$ for $40 \mathrm{sec}, 54{ }^{\circ} \mathrm{C}$ for $45 \mathrm{sec}$ and $72{ }^{\circ} \mathrm{C}$ for $1 \mathrm{~min}$, and a final extension of $72{ }^{\circ} \mathrm{C}$ for $10 \mathrm{~min}$. The PCR procedure for nLSU was as follows: initial denaturation at $94^{\circ} \mathrm{C}$ for $1 \mathrm{~min}$, followed by 35 cycles at $94^{\circ} \mathrm{C}$ for $1 \mathrm{~min}, 50{ }^{\circ} \mathrm{C}$ for $1 \mathrm{~min}$ and $72{ }^{\circ} \mathrm{C}$ for $1.5 \mathrm{~min}$, and a final extension of $72{ }^{\circ} \mathrm{C}$ for $10 \mathrm{~min}$. The PCR products were purified and sequenced in the Beijing Genomics Institute, China, with the same primers used in the PCR reactions.

\section{Phylogenetic analyses}

Phylogenetic analyses were applied to ITS + nLSU sequences. Sequences generated in this study were aligned with additional sequences downloaded from GenBank (Table 1) using Clustal X (Thompson et al. 1997) and manually adjusted in BioEdit (Hall 1999). Prior to phylogenetic analysis, ambiguous sequences at the start and the end were deleted and gaps were manually adjusted to optimize the alignment. Sequence alignment was deposited at TreeBase (submission ID 26119). Phylogenetic analysis was done as in Li et al. (2014) and Zhu et al. (2019). Sequences of Exidia candia Lloyd and Exidiopsis calcea (Pers.) K. Wells outside Hymenochaetales were used as outgroup referred to Miettinen and Larsson (2011) and Yuan et al. (2016), because some species related to Sidera in Polyporales, like Skeletocutis species, were added in phylogenetic analysis.

Maximum parsimony analysis (MP) was performed in PAUP* version 4.0b10 (Swofford 2002). All characters were equally weighted and gaps were treated as missing data. Trees were inferred using the heuristic search option with TBR branch swapping and 1000 random sequence additions. Max-trees were set to 1000 , branches of zero length were collapsed and all parsimonious trees were saved. Clade robustness (BP) was assessed using a bootstrap analysis with 1000 replicates (Felsenstein 1985).

The optimal substitution models for the combined dataset were determined using the Akaike Information Criterion (AIC) implemented in MrModeltest 2.2 (Nylander 2004) after scoring 24 models of evolution by PAUP* version 4.0 beta 10 (Swofford 2002). The selected model applied in the Bayesian phylogenetic inference (BI) analyses and Maximum likelihood (ML) analyses was the model GTR+I+G.

The BI analysis was performed with MrBayes 3.2.5 (Ronquist et al. 2012). Four Markov chains were run for 5 million generations and trees were sampled every 1000 
Table I. Information for the sequences used in this study.

\begin{tabular}{|c|c|c|c|c|}
\hline \multirow[t]{2}{*}{ Species } & \multirow[t]{2}{*}{ Specimen no. } & \multirow[t]{2}{*}{ Locality } & \multicolumn{2}{|c|}{ GenBank accession no. } \\
\hline & & & ITS & nLSU \\
\hline Ceriporiopsis aneirina & MUAF 888 & Czech Republic & EU340895 & EU340895 \\
\hline Contumyces rosella & Redhead 7501 & - & U66452 & U66452 \\
\hline Exidia candida & Spirin 8588 & USA & KY801870 & KY801895 \\
\hline Exidiopsis calcea & MW 331 & Canada & AF291280 & AF291326 \\
\hline Gloeoporus dichrous & KHL 11173 & Norway & EU118627 & EU118627 \\
\hline Gloeoporus hainanensis & Dai 15253 & China & KU360402 & KU360408 \\
\hline Globulicium hiemale & Hjm 19007 & Sweden & DQ873595 & DQ873595 \\
\hline Hyphodermella poroides & Dai 12045 & China & KX008367 & KX011852 \\
\hline Odonticium romellii & Murdoch 38 & Finland & MF319073 & MF318929 \\
\hline Oxyporus corticola & KHL 13217 & Estonia & DQ873641 & DQ873641 \\
\hline Phlebia georgica & KHL 12019 & Norway & DQ873645 & DQ873645 \\
\hline Repetobasidium conicum & KHL 12338 & USA & DQ873647 & DQ873647 \\
\hline Resinicium furfuraceum & KHL 11738 & Finland & DQ873648 & DQ873648 \\
\hline Rickenella mellea & Lamoure 74 & - & U66438 & U66438 \\
\hline Skvortzovia pinicola & KHL 12224 & USA & DQ873637 & DQ873637 \\
\hline Sidera lenis & Miettinen 11036 & Finland & FN907914 & FN907914 \\
\hline Sidera lowei & Miettinen X419 & Venezuela & FN907917 & FN907917 \\
\hline Sidera lunata & JS 15063 & Norway & DQ873593 & DQ873593 \\
\hline Sidera minutipora & Gates FF257 & Australia & FN907922 & FN907922 \\
\hline Sidera minutipora & Cui 16720 & Australia & MN621349 & MN621348 \\
\hline Sidera minutissima & Dai 19529 & Sri Lanka & MN621352 & MN621350 \\
\hline Sidera minutissima & Dai 19587 & Sri Lanka & - & MN621351 \\
\hline Sidera parallela & Cui 10346 & China & MK346145 & - \\
\hline Sidera parallela & Cui 10361 & China & MK346144 & - \\
\hline Sidera srilankensis & Dai 19581 & Sri Lanka & MN621345 & MN621347 \\
\hline Sidera srilankensis & Dai 19654 & Sri Lanka & MN621344 & MN621346 \\
\hline Sidera tenuis & Dai 18697 & Australia & MK331865 & MK331867 \\
\hline Sidera tenuis & Dai 18698 & Australia & MK331866 & MK331868 \\
\hline Sidera vesiculosa & BJFC025367 & Singapore & NH636565 & NH636567 \\
\hline Sidera vesiculosa & BJFC025377 & Singapore & NH636564 & NH636566 \\
\hline Sidera vulgaris & Ryvarden 37198 & New Zealand & FN907918 & FN907918 \\
\hline Skeletocutis amorpha & Miettinen 11038 & Finland & FN907913 & FN907913 \\
\hline Skeletocutis chrysella & Miettinen 9472 & Finland & FN907916 & FN907916 \\
\hline Skeletocutis lilacina & HHB 10522sp & USA & KY948834 & KY948894 \\
\hline Skeletocutis yuchengii & FBCC 1132 & China & KY953045 & KY953045 \\
\hline Skeletocutis yunnanensis & Dai 15709 & China & KU950434 & KU950436 \\
\hline Skeletocutis odora & L 13763sp & Canada & KY948830 & KY948893 \\
\hline Skeletocutis vulgaris & CBS 465.50 & France & MH856711 & - \\
\hline
\end{tabular}

New sequences are shown in bold.

generations. The first $25 \%$ of the sampled trees were discarded as burn-in, and the remaining ones were used to reconstruct a majority rule consensus tree and calculate Bayesian posterior probabilities (BPP) of the clades. The ML analysis was conducted on RAxmlGUI 1.31 (Michalak 2012), and all parameters used default settings. Statistical support values (BS) were obtained using non- parametric bootstrapping with 1000 replicates. The best fit maximum likelihood tree from all searches was kept. Branches that received bootstrap support values for MP and ML greater than or equal to $70 \%$ and BPP greater than or equal to 0.95 were considered as significantly supported. 


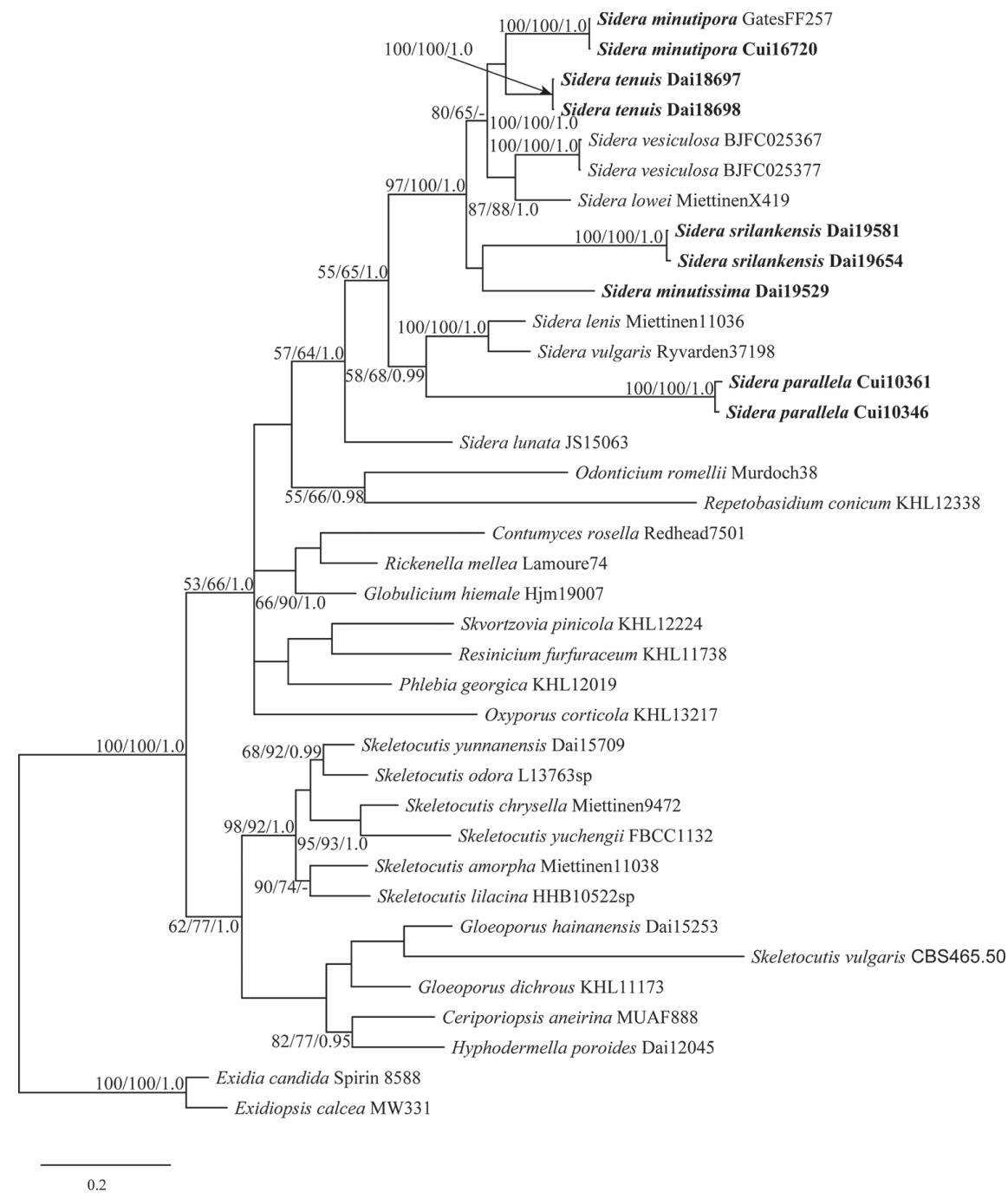

Figure I. Phylogeny of Sidera and related species generated by BI analysis based on combined ITS and nLSU sequences. Branches are labeled with MP and ML bootstrap values higher than 50\%, and BI probabilities more than 0.95 . New species and a new combination name are indicated in bold.

\section{Results}

\section{Phylogenetic analyses}

The combined ITS $+n L S U$ dataset included sequences from 37 specimens representing 32 species (Table 1). The specimen Dai 19587 was not included because of its lack of ITS sequence, but it has an nLSU sequence with $100 \%$ identity to Dai 19529 . The dataset had an aligned length of 1718 characters, of which 909 are constant, 148 are variable but parsimony-uninformative, and 661 are parsimony-informative. BI analyses resulted in a best tree (Figure 1), where the ESSs of all parameters were superior to 
1000 and the PSRFs were close to 1.0. MP and ML analyses produced consensus trees similar to $\mathrm{BI}$ analysis, and only the BI tree is presented along with support values from $\mathrm{MP}$ and ML analyses. Our newly generated sequences formed five robustly supported lineages within the Sidera clade, which we interpret as four new species and support for one new combination.

\section{Taxonomy}

Sidera minutipora (Rodway \& Cleland) Y.C. Dai, F. Wu, G.M. Gates \& Rui Du, comb. nov.

MycoBank No: 835373

Figures 2, 3

Poria minutipora Rodway \& Cleland, Pap. \& Proc. Roy. Soc. Tasmania 1929: 17 (1930). Basionym.

Type. Australia. New South Wales, Malanganee, 25 miles west of Casino, August 1917, MBT 35118.

Description. Basidiomata: Annual, resupinate, soft when fresh, soft corky to fragile when dry, up to $6.5 \mathrm{~cm}$ long, $3 \mathrm{~cm}$ wide, and approximately $1 \mathrm{~mm}$ thick at

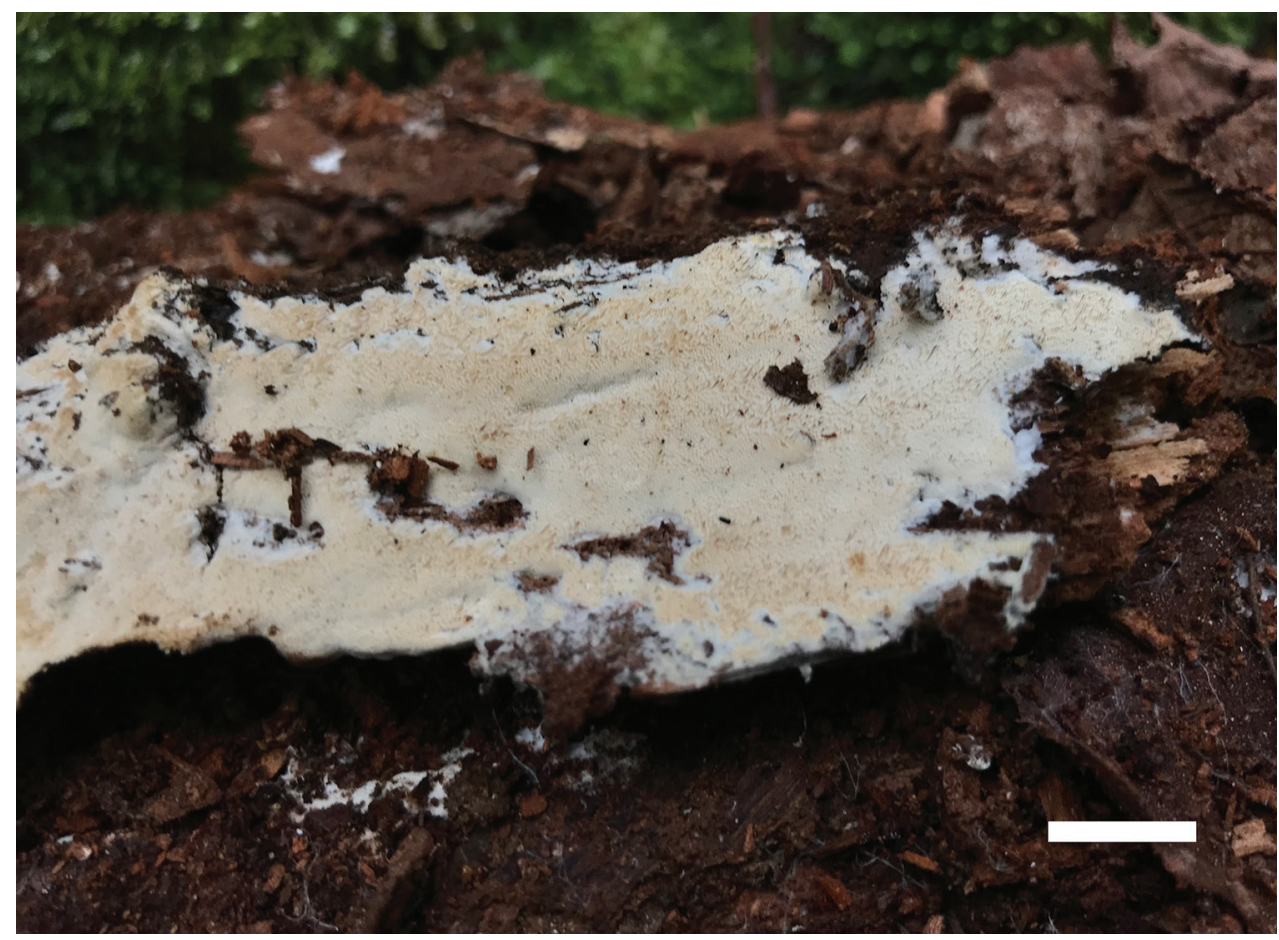

Figure 2. A basidioma of Sidera minutipora (Cui 16720). Scale bar: $1 \mathrm{~cm}$. Photo by Bao-Kai Cui. 
0. 6.80

08 \& 8 \&

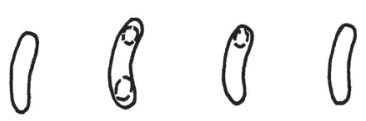
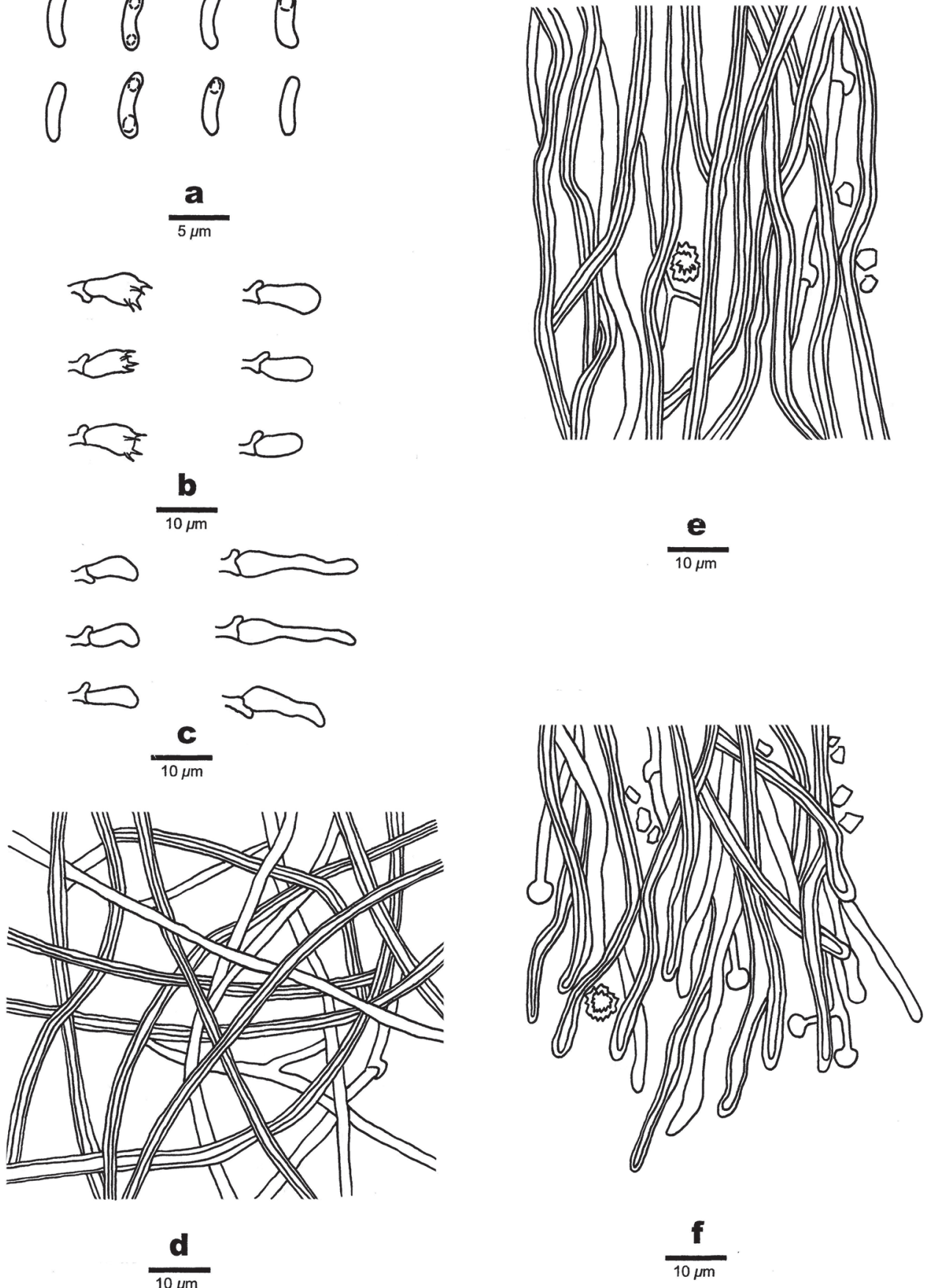

Figure 3. Microscopic structures of Sidera minutipora (Cui 16720) a basidiospores b basidia, basidioles $\mathbf{c}$ cystidioles $\mathbf{d}$ hyphae from subiculum $\mathbf{e}$ hyphae from trama $\mathbf{f}$ hyphae at dissepiment edge. Drawings by Rui Du. 
center; pore surface cream to buff when fresh, become buff to olivaceous buff when dry; sterile margin distinct, fimbriate, thinning out; pores round, 5-7 per $\mathrm{mm}$; dissepiments thin, lacerate; subiculum very thin to almost absent; tubes darker than the poroid surface, up to $1 \mathrm{~mm}$ long.

Hyphal structure: Hyphal system dimitic, generative hyphae bearing clamp connections; all hyphae IKI-, CB-, skeletal hyphae swolling in $\mathrm{KOH}$.

Subiculum: Generative hyphae hyaline, thin-walled, occasionally branched, 1-2 $\mu \mathrm{m}$ in diam; skeletal hyphae dominant, unbranched, interwoven, 1.5-2.5 $\mu \mathrm{m}$ diam; rosette-like crystals occasionally present, $1.5-7.0 \mu \mathrm{m}$ in diam, irregular crystals frequently present.

Tubes: Generative hyphae hyaline, thin-walled, occasionally branched, $1-2 \mu \mathrm{m}$ in diam, some with swollen tips; skeletal hyphae with a narrow lumen to subsolid, unbranched, interwoven, 1.8-3.0 $\mu \mathrm{m}$ diam; skeletal hyphae and generative hyphae co-dominating at dissepiment edges; rosette-like and irregular rhomboidal crystals occasionally present; cystidioles present, fusoid, hyaline, thin-walled, basally swollen, with a long or hyphoid neck, 7-19 $\times 2.4-4 \mu \mathrm{m}$; basidia barrel-shaped, hyaline, bearing four sterigmata and a basal clamp connection, $6.7-9 \times 3.5-4.5 \mu \mathrm{m}$; basidioles in shape similar to basidia, but slightly shorter.

Basidiospores: Allantoid, hyaline, thin-walled, smooth, occasionally with one or two guttules, IKI-, CB-, 3.7-4.3(-4.5) $\times 1-1.3 \mu \mathrm{m}, \mathrm{L}=4.01 \mu \mathrm{m}, \mathrm{W}=1.08 \mu \mathrm{m}$, $\mathrm{Q}=3.71(\mathrm{n}=30 / 1)$.

Specimen examined. Australia. Tasmania, Arve River Streamside Reserve, on rotten stump of Eucalyptus, 15 May 2018, B.K. Cui 16720 (BJFC 030019, Duplication in MEL); Warra LTER, 4305'4"S, 146 38'5"E, 16.Jan 2007 Gates FF257 (MEL).

\section{Sidera minutissima Y.C. Dai, F. Wu, G.M. Gates \& Rui Du, sp. nov.}

MycoBank No: 833182

Figures 4, 5

Type material. Holotype: SRI LANKA. Wadduwa, South Bolgoda Lake, on rotten angiosperm branch, 28 Feb 2019, Y.C. Dai 19529 (BJFC, isotype in University of Ruhuha).

Etymology. Minutissima (Lat.), refers to the species having small basidiomata.

Description. Basidiomata: Annual, resupinate, soft when fresh, soft corky to fragile when dry, up to $5 \mathrm{~cm}$ long, $3 \mathrm{~cm}$ wide, and approximately $1 \mathrm{~mm}$ thick at center; pore surface bluish to more or less turquoise when fresh, becoming cream to buff yellow when dry; sterile margin distinct, fimbriate, thinning out; pores round, 7-9 per $\mathrm{mm}$; dissepiments thin, entire; subiculum very thin to almost absent; tubes concolorous with pore surface, up to $1 \mathrm{~mm}$ long.

Hyphal structure: Hyphal system dimitic, generative hyphae bearing clamp connections; skeletal hyphae unbranched, interwoven, 2-3 $\mu \mathrm{m}$ diam; all hyphae IKI-, $\mathrm{CB}-$, unchanged in $\mathrm{KOH}$.

Subiculum: Generative hyphae hyaline, thin-walled, frequently branched, 1-2 $\mu \mathrm{m}$ in diam; skeletal hyphae dominant, more or less straight, unbranched, interwoven, 


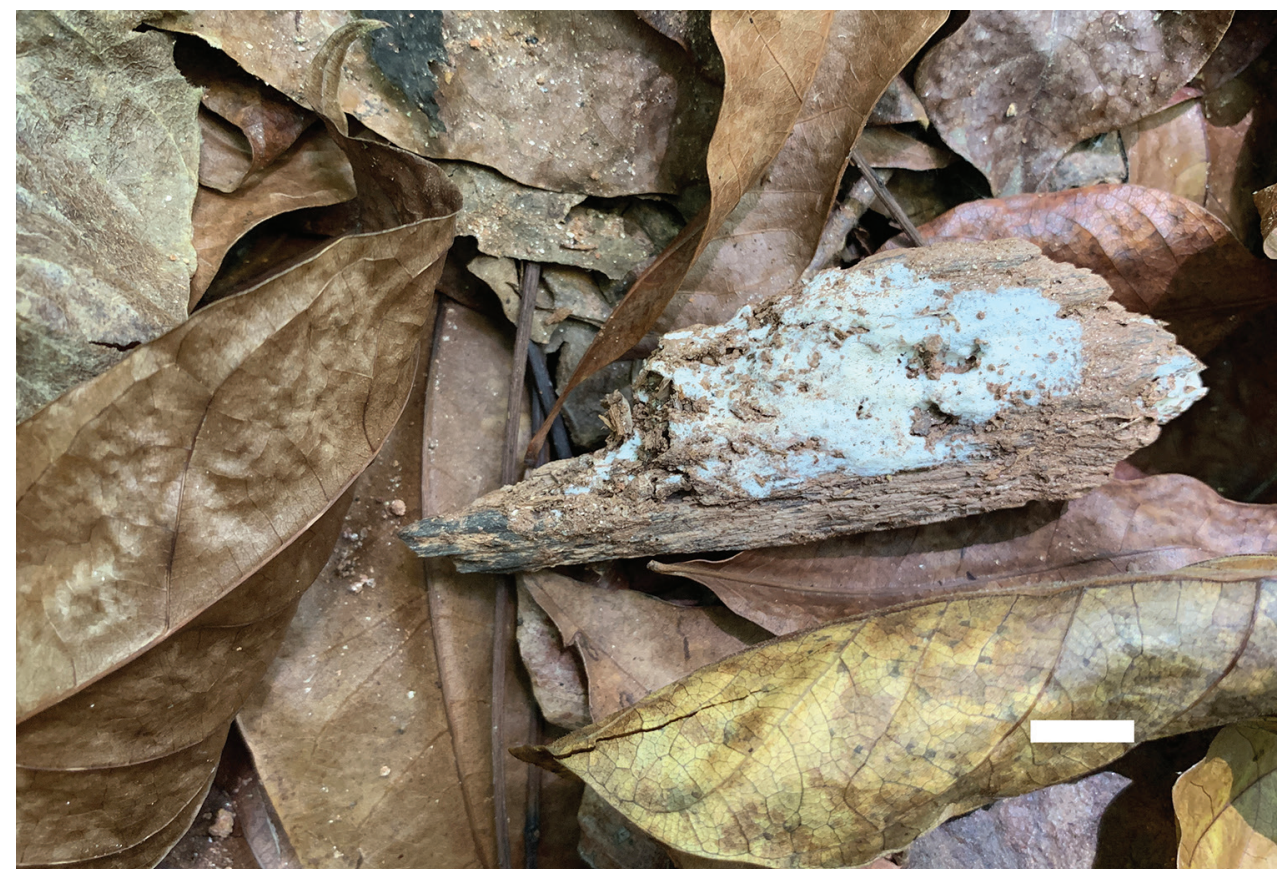

Figure 4. A basidioma of Sidera minutissima (paratype, Dai 19587). Scale bar: $1 \mathrm{~cm}$. Photo by Yu-Cheng Dai.

2-3 $\mu \mathrm{m}$ diam; rosette-like crystals frequently present, $2-8.5 \mu \mathrm{m}$ in diam, some irregular rhomboidal crystals present.

Tubes: Generative hyphae hyaline, thin-walled, frequently branched, $1-2 \mu \mathrm{m}$ in diam, some with swollen tips, dominating at dissepiment edges; skeletal hyphae with a narrow lumen to subsolid, unbranched, interwoven, 2-3 $\mu \mathrm{m}$ diam; rosette-like and irregular rhomboidal crystals abundant; cystidioles present, fusoid, hyaline, thin-walled, basally swollen, some with a long or hyphoid neck, $8-18 \times 2-5 \mu \mathrm{m}$; basidia barrelshaped, hyaline, bearing four sterigmata and a basal clamp connection, 7.1-12 × 3.5$4.8 \mu \mathrm{m}$; basidioles in shape similar to basidia, but slightly shorter.

Basidiospores: Allantoid, hyaline, thin-walled, smooth, occasionally with one or two guttules, IKI-, CB-, (3.7-)3.8-4.4(-4.5) $\times(0.8-) 0.9-1.3 \mu \mathrm{m}, \mathrm{L}=4.02 \mu \mathrm{m}$, $\mathrm{W}=1.07 \mu \mathrm{m}, \mathrm{Q}=3.67-3.85(\mathrm{n}=60 / 2)$.

Additional specimen examined (paratype). SRI LANKA. Kandy, Udawatta kele, Royal Forest Park. on rotten angiosperm wood, 2 Mar 2019, Y.C. Dai 19587 (BJFC).

\section{Sidera parallela Y.C. Dai, F. Wu, G.M. Gates \& Rui Du, sp. nov.}

MycoBank No: 829166

Figures 6, 7

Type material. Holotype: China. Yunnan Province, Lanping County, Luogujing Scenic Spot, on rotten angiosperm trunk, 19 Sep 2011, B.K. Cui 10346 (BJFC 011241). 


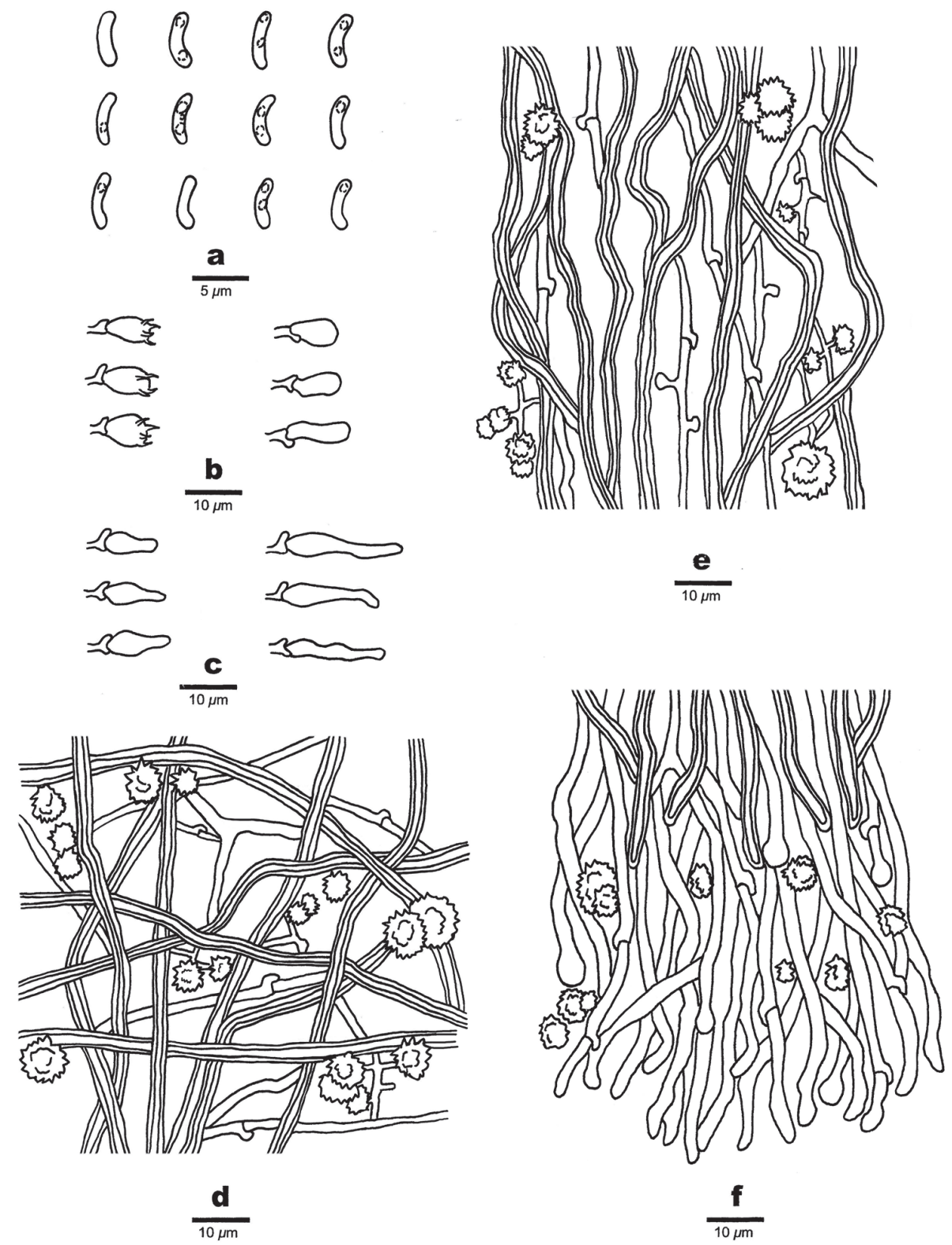

Figure 5. Microscopic structures of Sidera minutissima (holotype, Dai 19529) a basidiospores b basidia, basidioles $\mathbf{c}$ cystidioles $\mathbf{d}$ hyphae from subiculum e hyphae from trama $\mathbf{f}$ hyphae at dissepiment edge. Drawings by Rui Du.

Etymology. Parallela (Lat.), refers to the species having tubes with parallel tramal hyphae.

Description. Basidiomata: Annual, resupinate, soft corky when fresh, soft corky when dry, up to $11 \mathrm{~cm}$ long, $4 \mathrm{~cm}$ wide, and approximately $1.5 \mathrm{~mm}$ thick at center; 


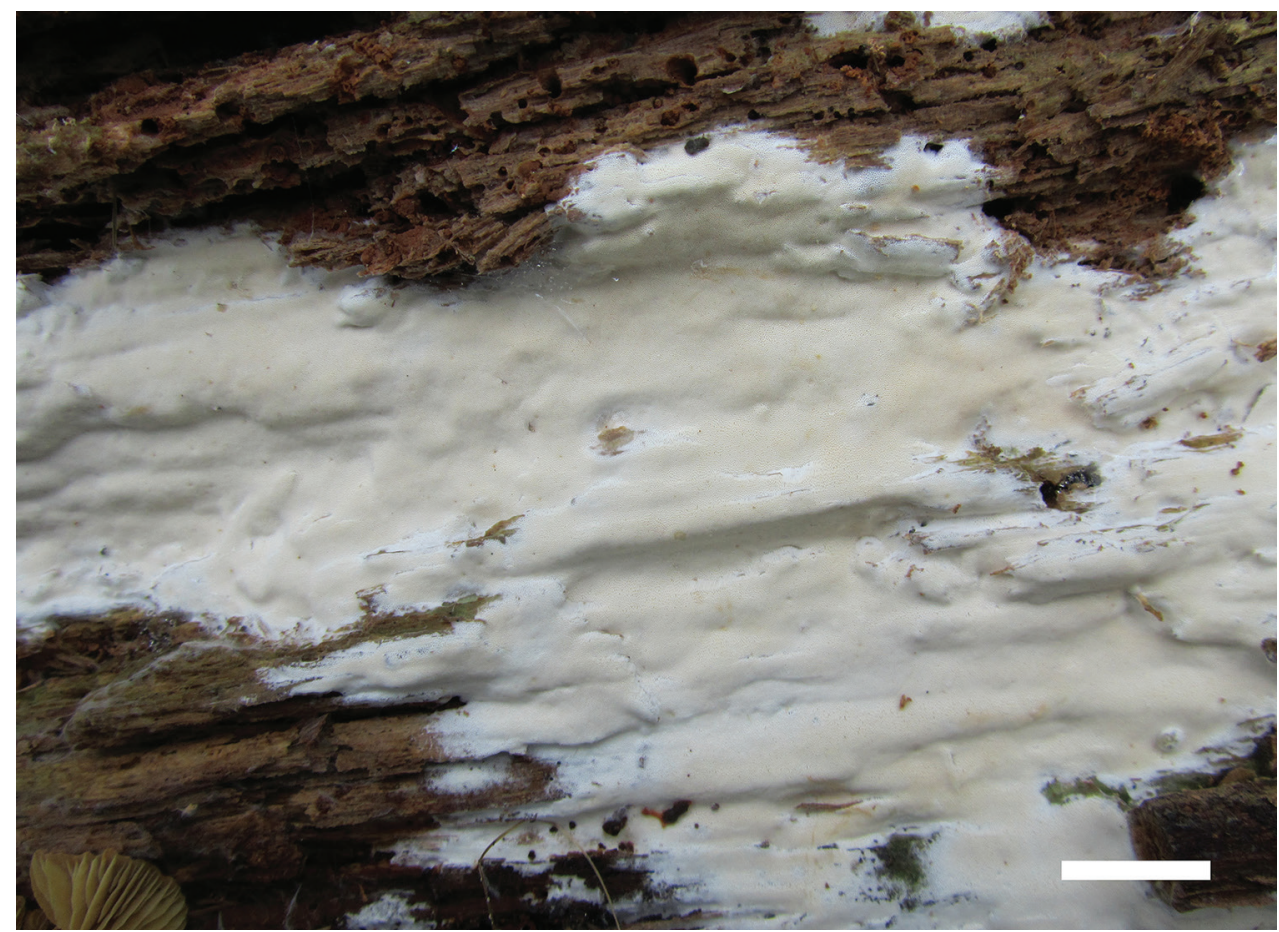

Figure 6. A basidioma of Sidera parallela (holotype, Cui 10346). Scale bar: $1 \mathrm{~cm}$. Photo by Bao-Kai Cui.

pore surface white when fresh, becoming cream to buff yellow upon drying; sterile margin distinct, fimbriate, thinning out; pores round, 6-8 per $\mathrm{mm}$; dissepiments thick, entire; subiculum very thin to almost absent; tubes concolorous with pore surface, up to $1.5 \mathrm{~mm}$ long.

Hyphal structure: Hyphal system dimitic, generative hyphae bearing clamp connections; skeletal hyphae dominant, unbranched, interwoven or parallel, 2-3 $\mu \mathrm{m}$ diam; all hyphae IKI-, CB-, unchanged in $\mathrm{KOH}$.

Subiculum: Generative hyphae hyaline, thin-walled, rarely branched, 1-2 $\mu \mathrm{m}$ in diam; skeletal hyphae dominating, more or less straight, unbranched, interwoven, 2-3 $\mu \mathrm{m}$ diam; rosette-like crystals frequently present, $2-8.5 \mu \mathrm{m}$ in diam, some irregular rhomboidal crystals present.

Tubes: Generative hyphae hyaline, thin-walled, rarely branched, 1-2 $\mu \mathrm{m}$ in diam, dominating at dissepiment edges; skeletal hyphae with a narrow lumen to subsolid, unbranched, parallel along the tubes, 2-3 $\mu \mathrm{m}$ diam; rosette-like and irregular rhomboidal crystals abundant; cystidia absent; cystidioles present, fusoid, hyaline, thin-walled, basally swollen, with a sharp or often hyphoid neck, 8.0-17 $\times 2.3-4 \mu \mathrm{m}$; basidia barrelshaped, hyaline, bearing four sterigmata and a basal clamp connection, 7-9 $\times 4-5 \mu \mathrm{m}$; basidioles in shape similar to basidia, but slightly shorter.

Basidiospores: Lunate, hyaline, thin-walled, smooth, occasionally with one or two guttules, IKI-, CB-, (2.7-)2.8-3.3 $\times(0.8-) 0.9-1.2 \mu \mathrm{m}, \mathrm{L}=3 \mu \mathrm{m}, \mathrm{W}=1.07 \mu \mathrm{m}$, $\mathrm{Q}=2.72-2.87(\mathrm{n}=60 / 2)$. 

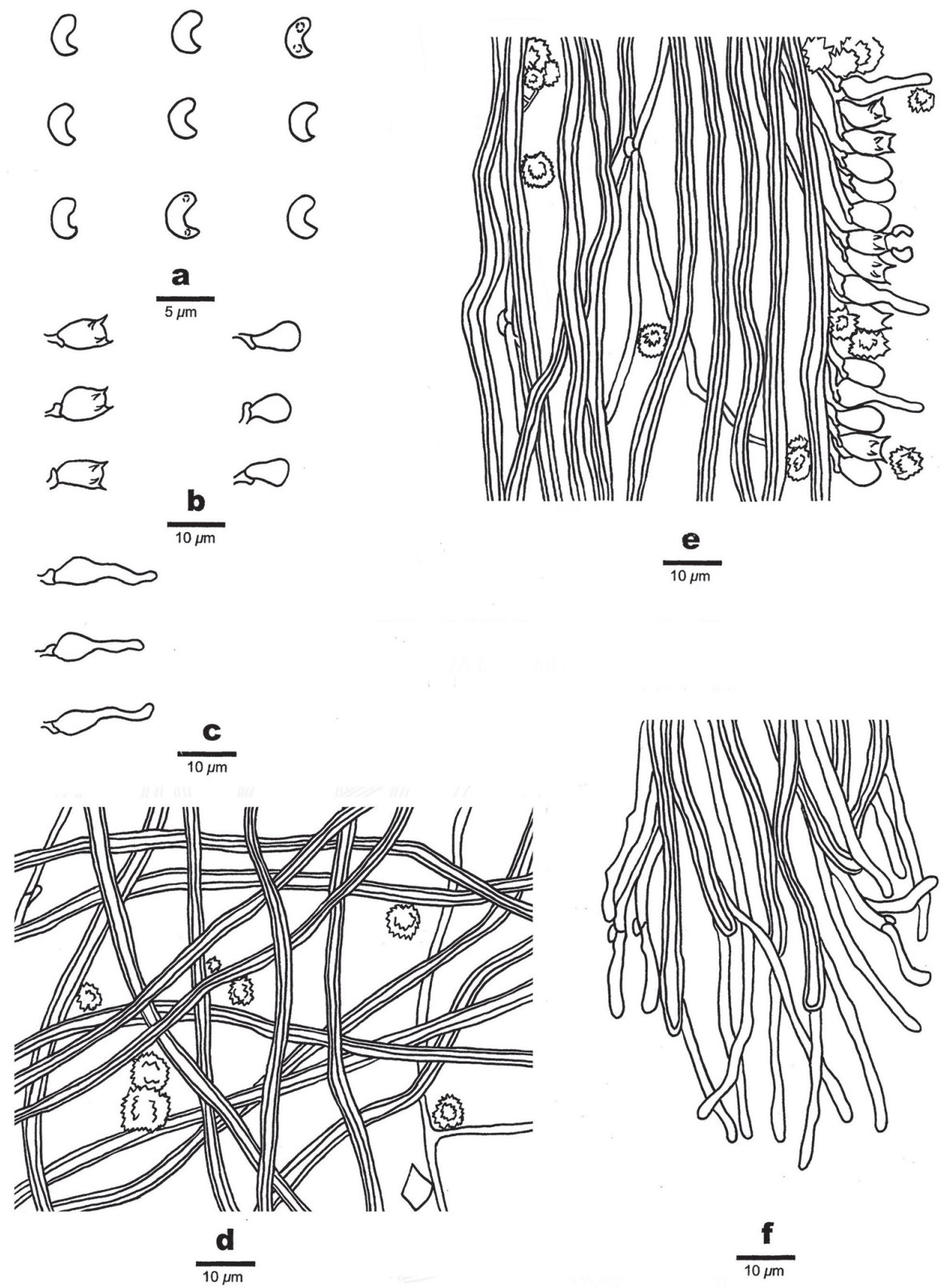

Figure 7. Microscopic structures of Sidera parallela (holotype, Cui 10346) a basidiospores b basidia, basidioles $\mathbf{c}$ cystidioles $\mathbf{d}$ hyphae from subiculum e hyphae from trama $\mathbf{f}$ hyphae at dissepiment edge. Drawings by Rui Du.

Additional specimen examined (paratype). China. Yunnan Province, Lanping County, Luogujing Scenic Spot, on fallen angiosperm trunk, 19 Sep 2011, B.K. Cui 10361 (BJFC 011256). 
Sidera srilankensis Y.C. Dai, F. Wu, G.M. Gates \& Rui Du, sp. nov. MycoBank No: 833183

Figures 8, 9

Type material. Holotype: SRI LANKA. Western Province, Mitirigala Nissarana, Vanaya Forest, on rotten angiosperm wood, 4 Mar 2019, Y.C. Dai 19654 (BJFC, isotype in University of Ruhuha).

Etymology. Srilankensis (Lat.), refers to the species being found in Sri Lanka.

Description. Basidiomata: Annual, resupinate, soft when fresh, soft corky to fragile when dry, up to $16.5 \mathrm{~cm}$ long, $3 \mathrm{~cm}$ wide, and approximately $1 \mathrm{~mm}$ thick at center; pore surface cream when fresh, becoming buff yellow upon drying; sterile margin distinct, fimbriate, thinning out; pores round, 6-8 per $\mathrm{mm}$; dissepiments thin, lacerate; subiculum very thin to almost absent; tubes concolorous with poroid surface, up to $1 \mathrm{~mm}$ long.

Hyphal structure: Hyphal system dimitic, generative hyphae bearing clamp connections; skeletal hyphae dominant, unbranched, interwoven, 1.5-3 $\mu \mathrm{m}$ diam; all hyphae IKI-, CB-, unchanged in $\mathrm{KOH}$.

Subiculum: Generative hyphae hyaline, thin-walled, frequently branched, 1-2 $\mu \mathrm{m}$ in diam; skeletal hyphae dominant, more or less straight, unbranched, interwoven, 1.5-3 $\mu \mathrm{m}$ diam; rosette-like crystals frequently present, 3.5-12 $\mu \mathrm{m}$ in diam, some irregular rhomboidal crystals present.

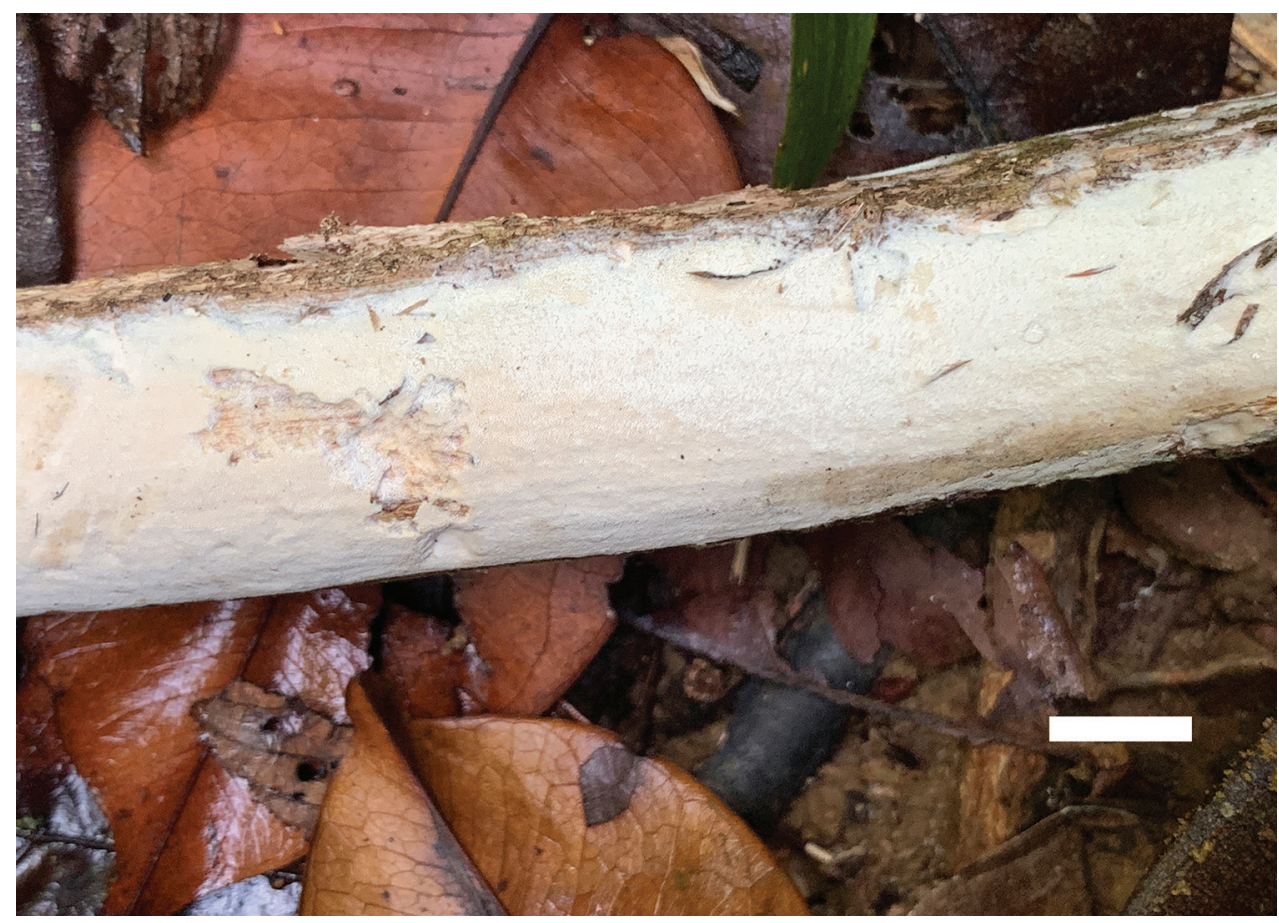

Figure 8. A basidioma of Sidera srilankensis (holotype, Dai 19654). Scale bar: $1 \mathrm{~cm}$. Photo by Yu-Cheng Dai. 

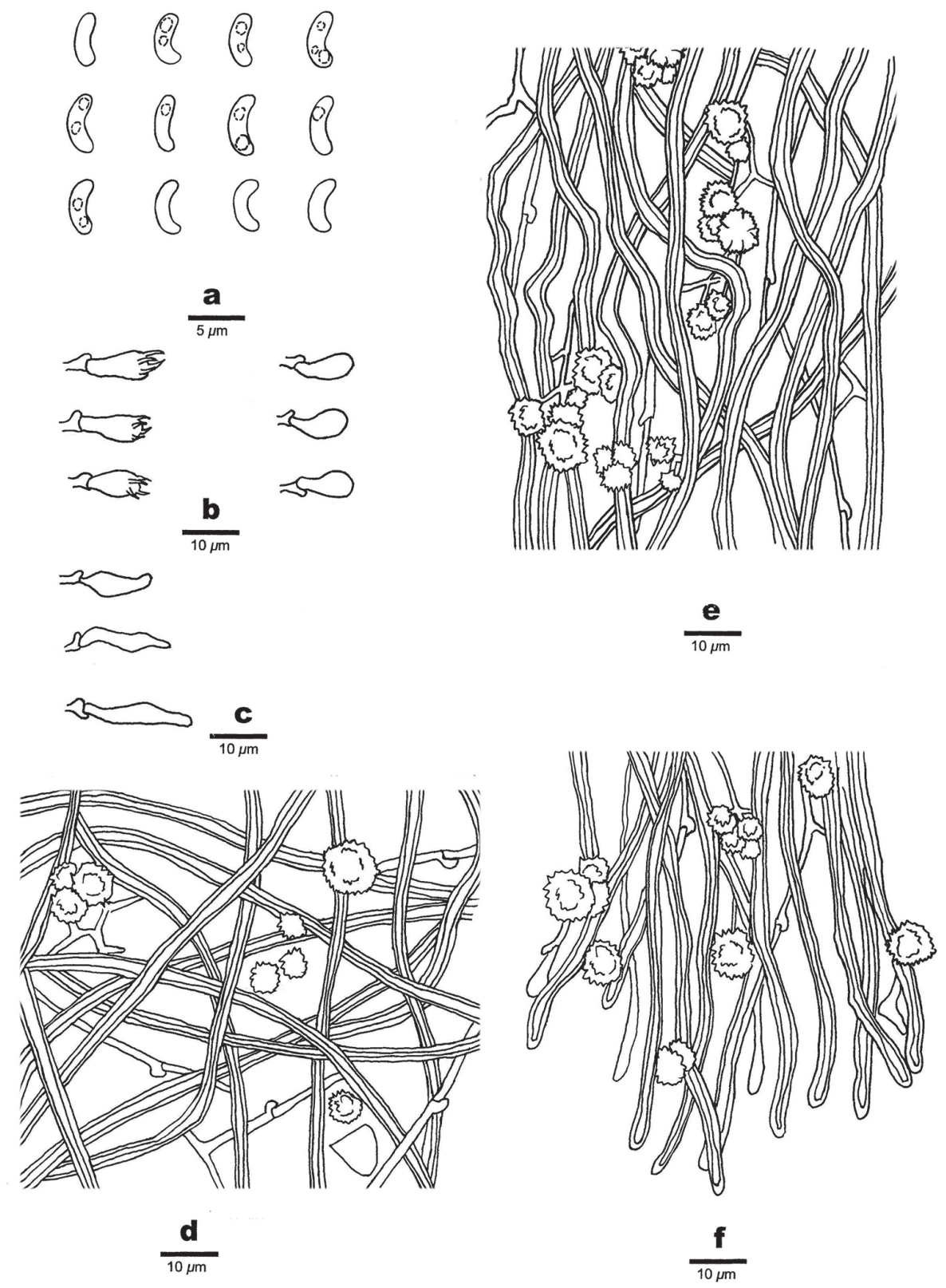

Figure 9. Microscopic structures of Sidera srilankensis (holotype, Dai 19654) a basidiospores b basidia, basidioles $\mathbf{c}$ cystidioles $\mathbf{d}$ hyphae from subiculum e hyphae from trama $\mathbf{f}$ hyphae at dissepiment edge. Drawings by Rui Du.

Tubes: Generative hyphae hyaline, thin-walled, frequently branched, $1-2 \mu \mathrm{m}$ in diam; skeletal hyphae with a narrow lumen to subsolid, unbranched, interwoven, 1.5$3 \mu \mathrm{m}$ diam; skeletal hyphae and generative hyphae co-dominating at dissepiment edges; rosette-like and irregular rhomboidal crystals abundant; cystidia absent; cystidioles 
present, fusoid, hyaline, thin-walled, basally swollen, with a sharp or often hyphoid neck, 8.1-14 × 3-4.1 $\mu \mathrm{m}$; basidia barrel-shaped, hyaline, bearing four sterigmata and a basal clamp connection, 7.8-13.2 × 3.6-4.5 $\mu \mathrm{m}$; basidioles in shape similar to basidia, but slightly shorter.

Basidiospores: Lunate, hyaline, thin-walled, smooth, occasionally with one or two guttules, IKI-, CB-, (3.4-)3.5-4(-4.1) x 1-1.3(-1.4) $\mu \mathrm{m}, \mathrm{L}=3.83 \mu \mathrm{m}, \mathrm{W}=1.16 \mu \mathrm{m}$, $\mathrm{Q}=3.28-2.34(\mathrm{n}=60 / 2)$.

Additional specimen examined (paratype). SRI LANKA. Kandy, Udawatta Kele, Royal Forest Park, on rotten angiosperm wood, 2 Mar 2019, Y.C. Dai 19581 (BJFC).

Sidera tenuis Y.C. Dai, F. Wu, G.M. Gates \& Rui Du, sp. nov.

MycoBank No: 829165

Figures 10, 11

Type material. Holotype: Australia. Tasmania, Hobart, Mt Wellington, on rotten wood of Eucalyptus, 13 May 2018, Y.C. Dai 18697 (BJFC 027166, isotype in MEL).

Etymology. Tenuis (Lat.), refers to the species having narrow basidiospores.

Description. Basidiomata: Annual, resupinate, soft and waxy when fresh, soft corky when dry, up to $10 \mathrm{~cm}$ long, $3 \mathrm{~cm}$ wide, and approximately $1 \mathrm{~mm}$ thick at cent-

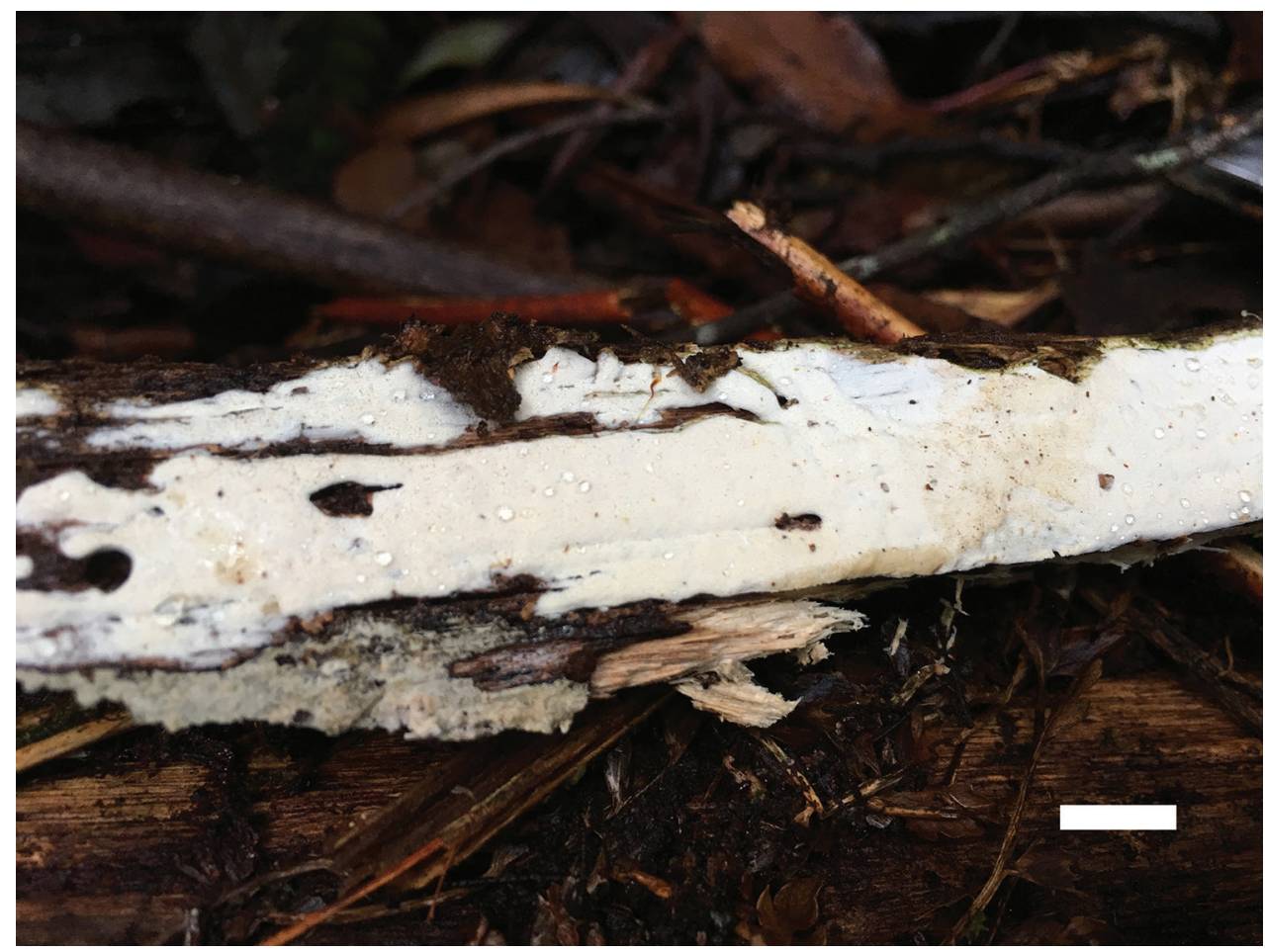

Figure 10. A basidioma of Sidera tenuis (holotype, Dai 18697). Scale bar: $1 \mathrm{~cm}$. Photo by Yu-Cheng Dai. 


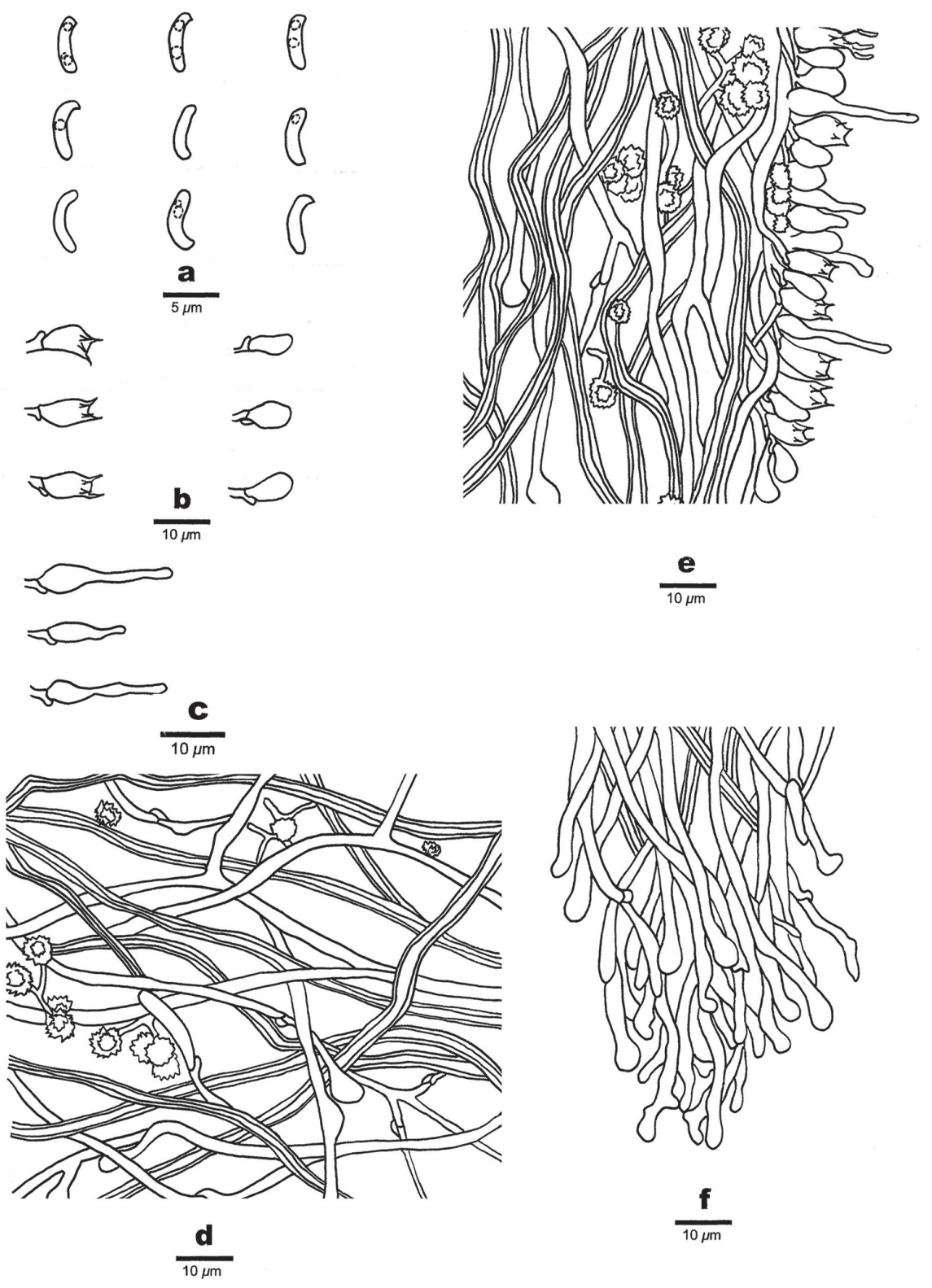

Figure I I. Microscopic structures of Sidera tenuis (holotype, Dai 18697) a basidiospores b basidia, basidioles $\mathbf{c}$ cystidioles $\mathbf{d}$ hyphae from subiculum e hyphae from trama $\mathbf{f}$ hyphae at dissepiment edge. Drawings by Rui Du.

er; pore surface white when fresh, becoming cream when dry; sterile margin indistinct; pores round, $8-10$ per $\mathrm{mm}$; dissepiments thin, entire; subiculum very thin to almost absent; tubes concolorous with poroid surface, up to $1 \mathrm{~mm}$ long. 
Hyphal structure: Hyphal system dimitic, generative hyphae bearing clamp connections; skeletal hyphae dominant, unbranched, interwoven, $2-3 \mu \mathrm{m}$ in diam; all hyphae IKI-, CB-, and unchanged in $\mathrm{KOH}$.

Subiculum: Generative hyphae hyaline, thin-walled, frequently branched, $1-2.5 \mu \mathrm{m}$ in diam, some with distinctly swollen tips which in shape are globose, bottle-shaped or irregularly elongated; skeletal hyphae dominant, unbranched, interwoven, $2-3 \mu \mathrm{m}$ in diam; rosette-like crystals frequently present, $2.5-10 \mu \mathrm{m}$ in diam, some irregular rhomboidal crystals present.

Tubes: Generative hyphae hyaline, thin-walled, frequently branched, 1-2.5 $\mu \mathrm{m}$ in diam, some with swollen tips, dominant at dissepiment edges; skeletal hyphae with a narrow lumen to subsolid, unbranched, interwoven, 2-3 $\mu \mathrm{m}$ diam; rosettelike and irregular rhomboidal crystals abundant; cystidia absent; cystidioles present, fusoid, hyaline, thin-walled, swollen at base, with a sharp or often hyphoid neck, 6-25 $\times 2.5-4.5 \mu \mathrm{m}$; basidia barrel-shaped, hyaline, bearing four sterigmata and a basal clamp connection, $7.3-11 \times 3.5-5 \mu \mathrm{dm}$; basidioles in shape similar to basidia, but slightly shorter.

Basidiospores: Allantoid, thin-walled, smooth, usually with one or two small guttules, IKI-, CB-, (4.1-)4.2-5(-5.4) $\times(0.7-) 0.8-1(-1.2) \mu \mathrm{m}, \mathrm{L}=4.62 \mu \mathrm{m}$, $\mathrm{W}=0.95 \mu \mathrm{m}, \mathrm{Q}=4.73-4.95(\mathrm{n}=60 / 2)$.

Additional specimen examined (paratype). Australia. Hobart, Mt Wellington, on rotten wood of Eucalyptus, 13 May 2018, Y.C. Dai 18698 (BJFC 027167).

\section{Key to species accepted in Sidera}

1 Hymenium grandinioid to odontioid. S. lunata

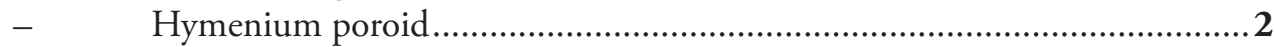

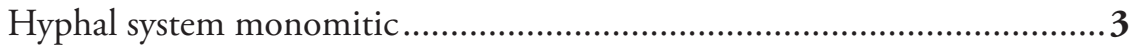

- $\quad$ Hyphal system dimitic .4

Basidiospores 2.9-3.7 × 0.6-1 $\mu \mathrm{m}$

S. vesiculosa

Basidiospores 3.5-5 × 1-1.2 $\mu \mathrm{m}$

S. lowei

Basidiomata perennial; basidiospores $>1.5 \mu \mathrm{m}$ in wildth S. lenis

Basidiomata annual; basidiospores $<1.5 \mu \mathrm{m}$ in wildth .5 Pore surface bluish when fresh S. minutissima

Pore surface white to cream or buff when fresh .6

Pores 5-8 per mm 7

Tramal hyphae parallel along tubes

S. parallela

Tramal hyphae interwoven in the tubes. 


\section{Discussion}

Previously five species of Sidera, viz. S. lenis, S. lowei, S. lunata, S. vesiculosa and S. vulgaris, were described or transferred to the genus. In this paper, Sidera minutissima, S. parallela, S. srilankensis and S. tenuis are described as new to science. In addition, Sidera minutipora is proposed as a new combination based on Poria minutipora. All these species with resupinate, white to cream or buff, bluish to more or less turquoise basidiomata when fresh, a dimitic hyphal system with generative hyphae bearing clamp connections, the presence of rosette-like crystals and allantoid to lunate basidiospores fit well in Sidera. Besides, they formed distinct lineages within the Sidera clade inferred from ITS and nLSU datasets (Figure 1).

Eight names were listed as synonyms of S. lenis (Index Fungorum and Mycobank): Poria lunulispora Pilát (type from Siberia), P. chakasskensis Pilát (type from Siberia), P. earlei Murrill (type from Jamaica), P. tenuipora Murrill (type from Jamaica), P. montana Murrill (type from Jamaica), P. consimilis Rick (type from Brazil), P. subvulgaris Rick (type from Brazil) and P. minutipora (type from Tasmania). Buchanan and Ryvarden (1993) indicated that the holotype of P. minutipora was not found, but an isotype PDD 7115 labelled part of type collection was studied. This comprised fragments of two species, Diplomitoporus lenis (P. Karst.) Gilb. \& Ryvarden (=Sidera lenis) and Schizopora flavipora (Berk. \& M.A. Curtis ex Cooke) Ryvarden [=Xylodon flaviporus (Berk. \& M.A. Curtis ex Cooke) Riebesehl \& Langer], and the portion of the isotype conforming to D. lenis was selected as lectotype for P. minutipora.

Three taxa were treated as synonyms of Sidera vulgaris (Index Fungorum and Mycobank): Boletus papyraceus Schrank, B. proteus Bolton and B. cellulosus O.F. Müll, and all of them were originally described from Europe, and they most probably represent a single species of S. vulgaris which was originally described from Sweden (Niemelä and Dai 1997).

In our phylogeny Gates FF257 clustered with Cui 16720 with high support within the Sidera clade (Figure 1), and both samples were collected from Tasmania, Australia. The sample Gates FF257 was named as S. vulgaris by Miettinen and Larsson (2011), but $S$. vulgaris was originally described from Europe and is different from the Australian specimens by having shorter basidiospores (2.9-3.6 $\times 0.9-1.4 \mu \mathrm{m}$ according to Niemelä and Dai 1997, vs. 3.7-4.3 $\times 1-1.3 \mu \mathrm{m}$ in Cui 16720). According to the protologue of Poria minutipora pores are 7 per $\mathrm{mm}$ and the only microscopic characteristic mentioned is that hyphae are 2-3 $\mu \mathrm{m}$ wide (Rodway and Cleland 1929). We did not study the type but our specimen Cui 16720 fits well with the description. Cunningham (1965) treated P. minutipora as a synonym of $P$. lenis (P. Karst.) Sacc. (=Sidera lenis), and indicated that spores were $2.5-4 \times 1-1.5 \mu \mathrm{m}$. Sidera tenuis is also described from Tasmania in the present paper, but differs from $S$. minutipora by smaller pores $(8-10$ per $\mathrm{mm})$ and longer basidiospores $(4.2-5 \times 0.8-1 \mu \mathrm{m})$. A European ITS sequence of Skeletocutis vulgaris (Fr.) Niemelä \& Y.C. Dai (ex. CBS 465.50 GenBank: MH856711.1) is close to Skeletocutis species and far from Sidera species in the phylogeny. Ryvarden 37198 from New Zealand also named as Sidera vulgaris by Miettinen and Larsson (2011) clustered with Sidera lenis from Finland with high support, but we didn't examine their morphology, thus we keep their name. 
Poria chakasskensis and P. lunulispora were described from Siberia (Pilát 1933, $1935)$ and both types were studied by Kotlaba and Pouzar $(1989,1991)$. The type of P. chakasskensis has basidiospores measuring 5.5-8.5 × 2-2.4 $\mu \mathrm{m}$ and represents Ceriporia purpurea (Fr.) Donk (Kotlaba and Pouzar 1991). Poria lunulispora was collected on wood of Pinus, and is true S. lenis (Kotlaba and Pouzar 1989, as Diplomitoporus lenis). Poria consimilis and P. subvulgaris were described from Brazil (Rick 1937). Rajchenberg (1987) studied the types and considered them as synonyms of S. lenis. Sidera lenis is a perennial species, and its basidiospores are more than $1.5 \mu \mathrm{m}$ wide. Our newly described species have annual basidiomata and basidiospores are less than $1.5 \mu \mathrm{m}$ wide. Poria earlei, P. montana and P. tenuipora were described from Jamaica (Murrill 1920a, b). Types of these species were studied by Niemelä and Dai (1997). They found that all types are sterile, but also that $P$. earlei and P. montana are conspecific and have perennial basidiomata, and that $P$. tenuipora has skeletal hyphae that are 3-4 $\mu \mathrm{m}$ in diam. Our new species are all annual and skeletal hyphae are $2-3 \mu \mathrm{m}$ in diam.

Phylogenetically, Sidera minutissima is closely related to S. vesiculosa, S. lowei, S. minutipora, S. tenuis and S. srilankensis (Fig. 1). However, S. vesiculosa and S. lowei are readily distinguished from $S$. minutissima by have a monomitic hyphal structure. S. minutipora, S. tenuis and S. srilankensis differ from S. minutissima by having white or cream pores when fresh. Sidera parallela is genetically close to S. lenis and S. vulgaris (Fig. 1), but $S$. lenis has perennial basidiomata and its basidiospores are more than $1.5 \mu \mathrm{m}$ wide, and S. vulgaris has interwoven tramal hyphae.

Morphologically Sidera minutipora resembles $S$. srilankensis by sharing similar size of pores and basidiospores. However, the former species has allantoid basidiospores, and its skeletal hyphae become swollen in $\mathrm{KOH}$ while basidiospores are lunate and skeletal hyphae are unchanged in $\mathrm{KOH}$ in S. srilankensis.

Sidera minutissima is similar to $S$. tenuis but differs by the bluish color of fresh basidiomata (white in $S$. tenuis) and by wider basidiospores $(0.9-1.3 \mu \mathrm{m}$ vs $0.8-1.0 \mu \mathrm{m}$ ).

Sidera parallela can be distinguished from other species by its parallel tramal hyphae. Sidera srilankensis resembles $S$. parallela by sharing pore size and lunate basidiospores, but in addition to the parallel tramal hyphae $S$. parallela also has smaller basidiospores measuring $2.8-3.3 \times 0.9-1.2 \mu \mathrm{m}$.

Sidera tenuis has the smallest pores of all species in the genus $(8-10$ per $\mathrm{mm})$ and also the narrowest basidiospores $(0.8-1 \mu \mathrm{m})$.

In this paper four new species and a new combination of Sidera are described from tropic and subtropic Asian-Pacific regions. Although the type species, Sidera lenis, has a distribution in boreal forests, the majority of species are so far found in tropical and subtropical regions.

\section{Acknowledgments}

The research is supported by the National Natural Science Foundation of China (Project Nos. U1802231, 31900019). 


\section{References}

Anonymous (1969) Flora of British Fungi. Colour Identification Chart. Her Majesty's Stationery Office, London.

Bourdot H, Galzin A (1928) Hyménomycètes de France. France, 764 pp.

Buchanan PK, Ryvarden L (1993) Type studies in the Polyporaceae 24. Species described by Cleland, Rodway and Cheel. Australian Systematic Botany 6: 215-235. https://doi. org/10.1071/SB9930215

Cunningham GH (1965) Polyporaceae of New Zealand. Bulletin of the New Zealand Department Scientific and Industrial Research 64: 1-304.

Cui BK, Li HJ, Ji X, Zhou JL, Song J, Si J, Yang ZL, Dai YC (2019) Species diversity, taxonomy and phylogeny of Polyporaceae (Basidiomycota) in China. Fungal Diversity 97: 137-392. https://doi.org/10.1007/s13225-019-00427-4

Dai YC (2010) Hymenochaetaceae (Basidiomycota) in China. Fungal Diversity 45: 131-343. https://doi.org/10.1007/s13225-010-0066-9

Dai YC, Yu CJ, Wang HC (2007) Polypores from eastern Xizang (Tibet), western China. Annales Botanicci Fennici 44: 135-145.

Du R, Wang L, Zhou M, Chen JJ (2019) A new species of Sidera (Hymenochaetales, Basidiomycota) from tropical Asia. Phytotaxa 387: 165-171. https://doi.org/10.11646/phytotaxa.387.2.9

Felsenstein J (1985) Confidence intervals on phylogenetics: an approach using bootstrap. Evolution 39: 783-791. https://doi.org/10.1111/j.1558-5646.1985.tb00420.x

Fries EM (1821) Systema Mycologicum. Berlingius lindae 1: 1-520.

Hall TA (1999) Bioedit: a user-friendly biological sequence alignment editor and analysis program for Windows 95/98/NT. Nucleic Acids Symposium Series 41: 95-98.

Li HJ, Cui BK, Dai YC (2014) Taxonomy and multi-gene phylogeny of Datronia (Polyporales, Basidiomycota). Persoonia 32: 170-182. https://doi.org/10.3767/003158514X681828

Kotlaba F, Pouzar Z (1989) Type studies of polypores described by A. Pilát 2. Ceská Mycology 43: 36-44.

Kotlaba F, Pouzar Z (1991) Type studies of polypores described by A. Pilát 4. Ceská Mycology 45: 91-97.

Michalak S (2012) RaxmlGUI: a graphical front-end for RAxML. Organisms Diversity \& Evolution 12: 335-337. https://doi.org/10.1007/s13127-011-0056-0

Miettinen O, Larsson KH (2011) Sidera, a new genus in Hymenochaetales with poroid and hydnoid species. Mycological Progress 10: 131-141. https://doi.org/10.1007/s11557-010-0682-5

Murrill WA (1920a) Light-colored resupinate polypores 1. Mycologia 12: 77-92. https://doi. org/10.1080/00275514.1920.12016821

Murrill WA (1920b) Light-colored resupinate polypores 2. Mycologia 12: 299-308. https://doi. org/10.1080/00275514.1920.12016844

Niemelä T, Dai YC (1997) Polypore Skeletocutis lenis and its sib S. vulgaris. Annales Botanici Fennici 34: 133-140.

Nylander JAA (2004) MrModeltest v2. Program distributed by the author. Evolutionary Biology Centre, Uppsala University, Uppsala. 
Petersen JH (1996) The Danish Mycological Society's colour-chart. Foreningen til Svampekundskabens Fremme, Greve.

Pilát A (1933) Additamenta ad floram Sibiriae Asiaeque orientalis mycologicam. Bulletin de la Société Mycologique de France 49: 256-339.

Pilát A (1935) Additamenta ad floram Sibiriae Asiaeque orientalis mycologicam. Pars tertia. Bulletin de la Société Mycologique de France. 51:351-426.

Rajchenberg M (1987) Type studies of Polyporaceae (Aphyllophorales) described by J. Rick. Nordic Journal of Botany 7: 553-568. https://doi.org/10.1111/j.1756-1051.1987.tb02023.x

Rick J (1937) Poriae Riograndenses. Brotéria Série Trimestral: Ciências Naturais 6: 128-152.

Rodway L, Cleland JB (1929) Notes on the genus Poria 3. Papers and Proceedings of the Royal Society of Tasmania 1929: 7-24.

Ronquist F, Teslenko M, van der Mark P, Avres DL, Darling A, Höhna S, Larget B, Liu L, Suchard MA, Huelsenbeck JP (2012) MrBayes3.2: efficient Bayesian phylogenetic inference and model choice, across a large model space. Systematic Biology 61: 539-542. https://doi.org/10.1093/sysbio/sys029

Shen LL, Wang M, Zhou JL, Xing JH, Cui BK, Dai YC (2019) Taxonomy and phylogeny of Postia. Multi-gene phylogeny and taxonomy of the brown-rot fungi: Postia and its related genera. Persoonia 42: 101-126. https://doi.org/10.3767/persoonia.2019.42.05

Swofford DL (2002) PAUP*: Phylogenetic analysis using parsimony (*and other methods). Version 4.0b10. Sinauer Associates, Sunderland. https://doi.org/10.1002/0471650129.dob0522

Thiers B (2018) Index Herbariorum: A global directory of public herbaria and associated staff. New York Botanical Garden's Virtual Herbarium, New York. http://sweetgum.nybg.org/science/ih/

Thompson JD, Gibson TJ, Plewniak F, Jeanmougin F, Higgins DG (1997) The CLUSTAL X windows interface: flexible strategies for multiple sequence alignment aided by quality analysis tools. Nucleic Acids Research 25: 4876-4882. https://doi.org/10.1093/nar/25.24.4876

White TJ, Bruns T, Lee S, Taylor J (1990) Amplification and direct sequencing of fungal ribosomal RNA genes for phylogenetics. In: Innis MA, Gefand DH, Sninsky JJ, White JT (Eds) PCR Protocols: A Guide to Methods and Applications. Academic Press, San Diego, 315-322. https://doi.org/10.1016/B978-0-12-372180-8.50042-1

Yuan HS, Dai YC (2008) Polypores from northern and central Yunnan Province, Southwestern China. Sydowia 60: 147-159.

Yuan Y, Ji XH, Wu F, He SH, Chen JJ (2016) Two new Gloeoporus (Polyporales, Basidiomycota) from tropical China. Nova Hedwigia 103: 169-183. https://doi.org/10.1127/nova_ hedwigia/2016/0344

Zhu L, Song J, Zhou JL, Si J, Cui BK (2019) Species diversity, phylogeny, divergence time and biogeography of the genus Sanghuangporus (Basidiomycota). Frontiers in Microbiology 10: 1-812. https://doi.org/10.3389/fmicb.2019.00812 